\title{
In vivo pharmacokinetics of levodopa and 3-O-methyldopa in muscle
}

\section{A microdialysis study}

\author{
Dirk Deleu $^{1}$, Sophie Sarre ${ }^{2}$, Guy Ebinger ${ }^{1}$, and Yvette Michotte ${ }^{2}$ \\ 1 Department of Neurology, University Hospital AZ-VUB, Vrije Universiteit Brussel, Laarbeeklaan 101 \\ 2 Department of Pharmaceutical Chemistry and Drug Analysis, Pharmaceutical Institute, Vrije Universiteit Brussel, \\ Laarbeeklaan 103, B-1090 Brussels, Belgium
}

Naunyn-Schmiedeberg's Arch Pharmacol (1991) 344:514-519

Unfortunaltely, two sentences in the Materials and methods section of this paper (p. 515) need correction. In the subsection "Instrumentation" the second sentence should read, "A chromatofield E201 amperometric detector (Chateaneuf-les-Martigues, France) was used." In the second paragraph of the subsection "Chromatographic conditions" the first sentence should read, "The detection limit was $5 \mathrm{pg}$ on the column for levodopa and $50 \mathrm{pg}$ on the column for 3-O-methyldopa." The retention time for levodopa was $8.67 \mathrm{~min}$ and for 3-O-methyldopa $18.33 \mathrm{~min}$. 